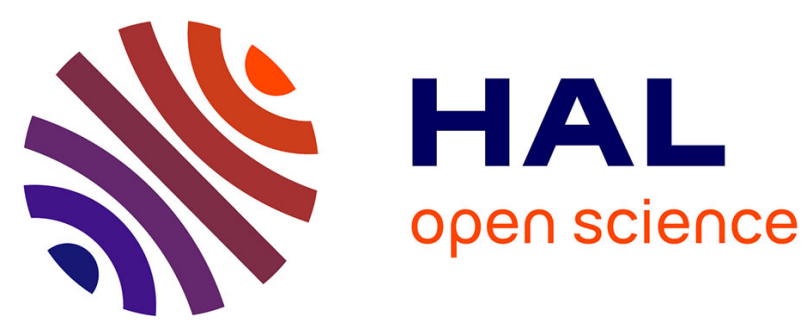

\title{
FEMMES ET IMAGES DE LUMIĖRE(S) : ÉMANCIPATION INTELLECTUELLE ET SENS DE LA VIE CHEZ MARY ASTELL
}

Guyonne Leduc

\section{> To cite this version:}

Guyonne Leduc. FEMMES ET IMAGES DE LUMIÈRE(S) : ÉMANCIPATION INTELLECTUELLE ET SENS DE LA VIE CHEZ MARY ASTELL. Bulletin de la Société d'Etudes Anglo-Américaines des XVIIème et XVIIIème siècles, 1999, 10.3406/xvii.1999.1428 . hal-03351991

\author{
HAL Id: hal-03351991 \\ https://hal.science/hal-03351991
}

Submitted on 22 Sep 2021

HAL is a multi-disciplinary open access archive for the deposit and dissemination of scientific research documents, whether they are published or not. The documents may come from teaching and research institutions in France or abroad, or from public or private research centers.
L'archive ouverte pluridisciplinaire HAL, est destinée au dépôt et à la diffusion de documents scientifiques de niveau recherche, publiés ou non, émanant des établissements d'enseignement et de recherche français ou étrangers, des laboratoires publics ou privés. 


\section{FEMMES ET IMAGES DE LUMIÈRE(S) : ÉMANCIPATION INTELLECTUELLE ET SENS DE LA VIE CHEZ MARY ASTELL}

[Fogs] Vanish before your Rising Sun of Wit. Like Stars, they only in themselves were bright, The whole Sex shines by your reflected Light. ${ }^{1}$

Bright Souls, they Eagle like did rise, Above the sight of vulgar Eyes. ...

What are those Souls which now like Diamonds shine? ${ }^{2}$

"Les Lumières," écrit Kant, "c'est pour l'homme sortir d'une minorité qui n'est imputable qu'à lui. La minorité, c'est l'incapacité de se servir de son entendement sans la tutelle d'un autre. . . . Aie le courage de te servir de ton propre entendement: telle est donc la devise des Lumières. ${ }^{33}$ En affirmant que tous les individus reçoivent des capacités intellectuelles égales, l'ère des Lumières s'avère une période essentielle pour la libération intellectuelle des femmes; si tous les esprits sont créés égaux et si la rationalité est la vertu principale, alors leur fonction procréatrice n'est plus un handicap pour elles. ${ }^{4}$ La nouvelle philosophie, que ce soit le

1. Judith Drake, "To the Most Ingenious Mrs--- on Her Admirable Defence of Her Sex" [by James Drake], An Essay in Defence of the Female Sex. In a Letter to a Lady. Written by a Lady (London, 1696) xii (12-14). Cet Essay n'est plus attribué à Astell: voir Florence Smith, Mary Astell (New York: Columbia UP, 1916) 173-82.

2. Mary Astell [1668-1731], "Judgment," "A Collection of Poems Humbly Presented and Dedicated to the Most Reverend Father in God William by Divine Providence Lord Archbishop of Canterbury," 1689, in Ruth Perry, The Celebrated Mary Astell: An Early English Feminist (Chicago: U of Chicago P, 1986) 416 (6.4-5), 419 (11.2).

3. Emmanuel Kant, "Réponse à la question: Qu'est-ce que les Lumières?", 1784, Qu'est-ce que les Lumières?, ed. and trans. Jean Mondot (Saint-Étienne: Publications de l'U de Saint-Étienne, 1991) 73.

4. Voir Hilda L. Smith, "'All Men and Both Sexes': Concepts of Men's Development, Women's Education, and Feminism in the Seventeenth Century," Man, God, and Nature in the Enlightenment, ed. Donald C. Mell (East Lansing: Colleagues P, 1988) 75-84. 
rationalisme cartésien ou l'empirisme baconien, souligne l'égalité originelle des hommes et des femmes dans la quête de la connaissance et de la vérité, et ne requiert que la capacité de penser avec logique.

La croyance de Mary Astell, élevée dans le giron de l'Église établie, en la raison et en un intellect asexué ainsi que sa foi en l'autonomie de l'esprit en font une "prophétesse de l'ère des Lumières." ${ }^{\text {I }}$ Consciente de l'impossibilité de modifier les conditions matérielles de leur existence, Astell veut inciter les femmes à une émancipation intellectuelle, morale et spirituelle. L'érudition importe moins que la capacité de raisonner jointe à la méthode pour apprendre à bien penser. Son propos est de libérer ses lectrices des fausses valeurs, de les amener à prendre conscience de leurs aptitudes et du véritable but de l'existence humaine: la recherche de la vérité immuable, accessible à tous les esprits qui s'y exercent. L'émancipation intellectuelle conduit précisément à la foi et à la compréhension de Dieu. À son sens, la finalité de la vie humaine est de contempler et d'aimer Dieu, la raison est une parcelle divine accordée à tout être humain et la cultiver est un acte religieux.

Influencée tant par le fond que par la forme du cartésianisme et du néoplatonisme, qui recourent à la lumière comme objet ou comme point de départ de comparaisons, ${ }^{6}$ de métaphores dont l'emploi est courant à la fin du XVIIe siècle, ${ }^{7}$ Astell tisse un réseau serré d'images liées à la lumière, présent sous divers aspects à tous les niveaux du dessein qui informe sa Serious Proposal to the Ladies for the Advancement of Their True and Greatest Interest, à savoir enseigner aux femmes à penser par elles-mêmes et à être des chrétiennes heureuses. ${ }^{8}$

5. Joan K. Kinnaird, "Mary Astell and the Conservative Contribution to English Feminism," JBS 19 (1979): 64 ("[a] prophetess of the Enlightenment"). Voir aussi Perry, "Radical Doubt and the Liberation of Women," ECS 18.4 (1985): 472-91 et Celebrated Mary Astell 70.

6. Voir Gérard Genette, "Le Jour, la nuit," Cahiers de l'Association Internationale des Études Françaises 20 (1968): 161 (ce que Greimas nommerait "l'isotopie du sémantisme lumineux").

7. La lumière renvoie à "une puissance de connaître, dont l'homme dispose et doit correctement user"; les lumières désignent des "connaissances, dont on indique la source" (les lumières de la foi, de la raison, par exemple); voir Jacques Roger, "La Lumière et les Lumières," Cahiers 168.

8. La Proposal fut publiée en deux parties indépendantes (1694, 1697); la seconde a pour sous-titre "Wherein a Method Is Offer'd for the Improvement of Their Minds." Lors de sa publication anonyme, la Proposal fut attribuée à Damaris Cudworth Masham; sur ses liens intellectuels avec Astell et Norris, voir Patricia Springborg, "Astell, Masham, and Locke: Religion and Politics," Women's Writers and the Early Modern British Political Tradition, ed. Smith (Cambridge: Cambridge UP, 1998) 105-25. Astell consacre 
La dynamique de l'image omniprésente de la lumière et le mécanisme des métaphores s'observent dans l'association des femmes et de la lumière - les femmes pourraient irradier une lumière plus belle -, dans le traitement que réserve Astell au véritable but de l'existence humaine - à la fois recherche de la vérité et amour de Dieu -, et à travers l'écriture même de sa pensée, vecteur de ses conseils aux femmes, où la clarté est le maîtremot.

L'association entre femmes et lumière est récurrente sous la plume d'Astell. Elles brillent tel un réceptacle ou telle une source de lumière dont la nature émane soit de l'extérieur, soit de l'intérieur. L'auteur recourt à la métaphore banale de l'éclat de la beauté physique qui rayonne autour de la femme: "your Beauty casts a lustre round about." ${ }^{9}$ Cependant, comme les adjectifs épithètes "corruptible" et "immortal" (1.5) viennent de souligner les essences opposées du corps et de l'âme et qu'Astell compare ensuite ce rayonnement physique à celui, plus grand, de la beauté de l'âme ("infinitely more bright and radiant" [7]), sa technique de présentation permet de démontrer que la seule vraie beauté est intérieure. La suite de la comparaison le confirme en exposant que beauté extérieure et beauté intérieure peuvent se contrebalancer: les femmes peu gâtées par la Nature ont la possibilité de compenser leur disgrâce par une beauté intérieure, démarche accessible aussi aux femmes belles, dont la beauté extérieure, sinon, peut être ternie par une laideur morale ("tarnish" est utilisé aux sens propre et figuré) (5). Astell va s'employer à cerner et l'origine et la nature de cette seule vraie beauté.

Cette source intérieure de lumière se trouve mentionnée, mais non définie, dans la Partie 1: "that particle of Divinity" (6); opposée au corps mortel, cette "particule" désigne l'âme, déduction vérifiée dès la page suivante (7). Dans la Partie 2, elle s'intellectualise: "this bright side of us" (99) s'applique à "our Understanding" (99) puis à la raison individuelle ("the Divine Reason of which ours is but a short faint Ray" [105]). Dès lors, on note un transfert dans la métaphore; ce n'est plus la femme, réceptrice de cette lumière envoyée par Dieu, qui brille, mais directement sa raison $(107,108)$. Astell reprend la comparaison de l'être humain comme réceptacle de l'étincelle divine qu'est la raison, source du rayonnement intérieur. Une transition s'effectue entre âme et raison; principe

un autre ouvrage aux femmes: Some Reflections upon Marriage (1700) qui dénonce le déséquilibre des relations de pouvoir dans le mariage.

9. Astell, A Serious Proposal to the Ladies, for the Advancement of Their True and Greatest Interest. By a Lover of Her Sex, 1694, 1697, ed. Springborg (London: Pickering, 1997) 1.7 (pour Partie 1, page 7). 
spirituel et principe rationnel sont réunis dans l'expression "Humane Nature consists of the Union of a Rational Soul with a Mortal Body" (157-58) où, par symétrie, "rational" équivaut à "immortal."

Leur coexistence s'explique par le contexte théologico-philosophique, par la conception contemporaine de la raison notamment. D'une part, Astell est influencée par le refus de Whichcote, platonicien de Cambridge, d'opposer spirituel et rationnel, surnaturel et naturel, Grâce et Nature: "I oppose not rational to spiritual; for spiritual is most rational."10 Tel est pour Constantinos A. Patrides "one of the boldest challenges to the modes of thought characteristic of traditional Protestantism."11 Dans ce sillage, elle fournit une définition de la raison et de la révélation: "Revelation ... is but an exaltation and improvement of Reason" (2.100), idée qu'elle développe plus loin (156). ${ }^{12}$ D'autre part, Astell subit l'emprise de Descartes, qui appelle "lumière naturelle" la "faculté de connaitre" que Dieu "nous a donnée,"13 et de la tradition des Pères de l'Église et des théologiens du Moyen Âge, qui trouve ses racines dans le texte du Prologue de l'Évangile de saint Jean $(1.4,5,9)$ et chez saint Augustin. Malebranche, qu'elle connaît aussi, tend, écrit Jacques Roger, à confondre "le mouvement de la lumière révélée et de la lumière naturelle . . . qui n'est plus semence déposée en l'homme mais émanation, effusion de la lumière divine."14 Révélée ou naturelle, "la lumière est toujours émanation de l'Absolu, don gratuit de Dieu." ${ }^{15} \mathrm{La}$ lumière de la révélation est issue de l'Écriture et la lumière naturelle, de même essence divine, se trouve en l'homme.

Astell emprunte à Ficin l'image du rayon divin: "our care then must be to open our Eyes to that Beam of Light [Reason]" (2.108). Selon le

10. Benjamin Whichcote, Eight Letters of Dr. Antony Tuckney, and Dr. Benjamin Whichcote . . . Written in September and October, MDCLI, appended to Moral and Religious Aphorisms . . . Published in MDCCIII, by Dr Jeffery. Now Republished, with Very Large Additions, by Samuel Salter (London, 1753) 108.

11. Voir Constantinos A. Patrides, ed., The Cambridge Platonists (London: Arnold, 1969) 10.

12. "the Eternal Word and Wisdom of GOD declares his Fathers Will unto us, by Reason which is that Natural and Ordinary Revelation by which he speaks to every one; and by that which is call'd Revelation in a stricter Sense, which is nothing else but a more perfect and infallible way of Reasoning, whereby we are Clearly and Fully instructed in so much of GOD's Will as is fit for us to know" (2.156).

13. René Descartes, Principes de la philosophie, 1644, Descartes. Euvres et lettres, ed. André Bridoux (Paris: Gallimard, "Bibliothèque de La Pléiade," 1949) 446 (Partie 1, $\S 30)$.

14. Roger 169.

15. Roger 168. 
platonicien florentin, l'âme reçoit de Dieu un "ray divin." ${ }^{16}$ En revanche, elle néglige l'expression phare des platoniciens de Cambridge influencés par Ficin: "the spirit of man is the candle of the Lord" (Ps. 20.27). ${ }^{17}$ Leur interprétation diffère de celle, habituelle, des protestants, rappelle Patrides: "Protestants normally deployed the metaphor in order to emphasise the inadequacy of natural knowledge. . . ." ${ }^{18}$ Ils ne rejettent pas la définition classique de la raison comme intellection, comme faculté raisonnante; sous l'influence de Whichcote surtout, "the candle of the Lord" désigne une "faculté de l'esprit."19 Cette "bougie" n'est pas un simple don de la grâce divine à certains élus ou une lumière intérieure incertaine; c'est la recta ratio de la tradition humaniste; elle est là où se trouve la vera fides. ${ }^{20}$ Aucun conflit, pour lui, entre raison et foi. La raison n'est pas simplement une faculté critique et logique, mais la faculté platonicienne capable d'atteindre la vérité divine. Pour Astell, à l'instar des platoniciens chrétiens, il s'agit bien d'une parcelle divine; en outre, puisque Dieu est lumière, cette particule divine l'est ipso facto.

Au début de la Proposal, Astell joue donc sur la vanité féminine et adopte une logique du superficiel destinée à l'exploiter dans sa propre optique: développer la beauté immatérielle qui éclipsera la beauté physique puis, à terme, la remplacera. À cette fin, elle utilise la valeur ambivalente que représente le corps. Pour en rappeler l'aspect positif, l'auteur emprunte les deux métaphores chrétiennes du corps, temple ("those Glorious Temples" [1.7]) et tabernacle ("our Earthly Tabernacle" [2.160]), donné par Dieu et qu'à ce titre il faut entretenir (1.45), soulignet-elle en y ajoutant la dimension religieuse (2.151). Plus souvent, elle en évoque le versant négatif à travers l'image platonicienne, reprise par les platoniciens chrétiens, de l'âme emprisonnée dans une enveloppe de limon: "these Mud-walls that enclose our Earthly Tabernacle" (2.160). Astell s'applique, dès le départ, à marteler avec réalisme l'antinomie entre

16. Voir Robert Ellrodt, Neoplatonism in the Poetry of Spenser (Genève: Droz, 1960) 133 (n. 68). L'influence de platoniciens chrétiens de l'Académie de Florence (Marcile Ficin et Pic de la Mirandole) s'exerça surtout sur les platoniciens de Cambridge, notamment sur John Norris, correspondant d'Astell.

17. Voir chez Whichcote, Aphorisms $§ 916$ ("The Spirit of a Man is the Candle of the Lord; Lighted by God, and Lighting us to God. Res illuminata, illuminans") et The Use of Reason in Matters of Religion, Select Sermons, ed. Anthony Ashley Cooper (London, 1698) 449 ("A Candle lighted by God, and serving to this Purpose; to discover and discern God").

18. Patrides 11.

19. Voir Patrides 12, 13 ("a spiritual faculty, as that power of mind which the Areopagite termed 'the union above mind' ... and which Plotinus described as 'something greater than reason' . . . Henry More's term for this faculty was 'Divine Sagacity"').

20. Voir Whichcote, Letters 108. 
éphémère et durable. La beauté physique est liée à la mort (1.5) par le biais de deux processus de dégradation - réduction du corps en "poussière" (6) et "putréfaction" (7). ${ }^{21}$ Ce substantif s'intègre dans une phrase aux connotations dépréciatives qu'exprime le choix d'adjectifs liés par une allitération et emblématiques d'un caractère trompeur et racoleur: ${ }^{22}$ "I cannot . . . reflect, that those Glorious Temples . . . be like a garnish'd Sepulchre, which for all its glittering, has nothing within but Emptiness and Putrefaction" (7).

Pour forcer le trait, originalité, vivacité et puissance se conjuguent dans la métaphore immobilière qui contraste location et château: le corps est une location où l'âme dispose d'un bail court alors que la demeure divine est éternelle (1.14). Est ici reprise l'image du château: "one of the highest Mansions in the Court of Heav'n" (7). ${ }^{23}$ Dans ce contexte, il importe de remettre le corps à sa place toute relative par rapport à l'âme, ce que souligne la comparaison: "Tis a Godlike thing . . to keep a Body from perishing, but it is much more Divine, to Save a Soul from Death!" (2.151). La lumière viendra récompenser ceux/celles qui s'y efforceront: "They . . . shall shine as the brightness of the Firmament, and they who turn many to Righteousness as the Stars for ever" (151). ${ }^{24}$ Alors, les femmes brilleront d'une lumière authentique.

D'autres raisons expliquent que les femmes sont source de lumière ou qu'elles la réfléchissent. Tout d'abord, elles peuvent rayonner par leur exemplarité dans le domaine religieux; les adjectifs qualificatifs récurrents sont alors "bright" et "illustrious," renforcés par un superlatif ("the brightest Patterns of Religion" [1.9] et "that holy emulation which a continual view of the brightest and most exemplary Lives will excite in us" [35]) ou par un adverbe ("such illustrious patterns" [9]). Ces adjectifs permettent de rappeler l'importance et la responsabilité de la classe supérieure qui (se) doit (de) servir de modèle: "if our Example be Bright it becomes the more Illustrious ..." (2.174). Un glissement s'opère de la personne à son exemple, puis un autre de la source lumineuse à la cible éclairée dans "what a blessed World shou'd we have, shining with so

21. La structure de la Partie 1 est très serrée; à preuve, la reprise de ces deux éléments à la fin (46); le terme "Carcass" $(26,147)$ est ajouté.

22. "To Garnish" signifie "[to] decorate, embellish for purpose of attaching money belonging to debtor" et le $2^{\mathrm{e}}$ sens de "to glitter" est "[to] be showy or splendid" (OED). En arrière-plan, se profilent les opposés que sont le Veau d'or vétérotestamentaire (Dan. 3.714, 2 Rois 10.29) et le saint sépulcre néotestamentaire.

23. Le lexique de la construction rappelle le Nouveau Testament (Jean 14.2). "Mansion" (aussi 1.26) se trouve, avec cohérence, dans son poème "Ambition": "a higher Mansion there" (Perry, Celebrated Mary Astell 405).

24. Astell cite, en italiques, Daniel (12.3). 
many stars of Vertue!" (1.38); par osmose, le monde s'illuminerait grâce à leur exemple fondé sur leurs actes. Pour les platoniciens de Cambridge dont Whichcote, ${ }^{25}$ les bonnes actions nourrissent toute vie chrétienne; à leur exemple, Astell encourage ses consœurs à traduire concrètement leurs bons sentiments (2.72). Les auteurs seront récompensés de leurs actes, écrit-elle en puisant chez Matthieu (6.19-20): "to amass huge Treasures of good Works, and to procure one of the brightest Crowns of Glory" (1.20). L'intensité de la lumière devient élévation morale dans "Acts of Beneficence, Liberality and Charity, are full of Lustre, they procure for their Possessor a lofty Character" (2.173).

La lumière diffusée par les femmes est, d'autre part, associée à leur vertu $(1.38,2.174)$ et opposée à l'obscurité du vice qu'elle peut vaincre (1.9). Certains passages relient "vertue" et "illustrious" (1.69) ou "bright" (2.74). Ce dernier terme réapparaît lorsque, par déplacement, la vertu elle-même brille ("Vertue her self as bright as she is" [1.44]) et illumine le visage féminin ("will shine thro' and brighten your very Countenance" [45]). La beauté de la vertu la rend aimable et engage à la pratiquer (2.74); Astell entend ce terme au sens spirituel. ${ }^{26}$

Outre leur vertu, leur intelligence, où réside la véritable valeur, peut être, chez les femmes, source de lumière: "This [mind] is the richest Ornament, and renders a Woman glorious in the lowest Fortune: So shining is real worth, that like a Diamond it loses not its lustre, tho' cast on a Dunghill" (1.46). Les lectrices ne peuvent qu'être sensibles à la métaphore de la parure puis à la comparaison avec le diamant, symbole de perfection, d'incorruptibilité, de réalité absolue et de l'amour divin (dio-amando).

Cette lumière revêt diverses formes flatteuses, païennes et chrétiennes. Le rayonnement des femmes est assimilé à celui des étoiles, dont on retient la qualité de luminaire $(1.38,2.151)$, et désigné par son intensité et par son ampleur $(1.45){ }^{27}$ La lumière diffusée forme un halo $(1.38,45)$ autour de la femme qui brille elle-même (2.88) ou par l'intermédiaire de ses rayons nommés explicitement: "we never shine so Illustriously as when we break thro these little Clouds ... which impotently attempted to obscure our Rays" (2.95). Point commun, le verbe "to shine," normale-

25. Voir "I act, therefore I am" (Whichcote, Several Discourses, ed. John Jeffery, 4 vols. [London, 1701-1707] 2: 135, 3: 328).

26. "The due Performance of which is what we call Vertue, which consists in governing Animal Impressions, in directing our Passions to such Objects, and keeping 'em in such a pitch, as right Reasons require" (2.161).

27. Dans 2.151, elle cite Daniel 12.3. Peut-être songe-t-elle à l'apparition, chez lui, de l'image de l'étoile. 
ment spécifique du soleil, facilite le passage graduel à l'évocation de l'astre, aux rayons duquel sont comparés les arguments des femmes (150). Le cercle projeté autour de la femme-étoile ou de la femme-soleil permet de glisser à la couronne, empruntée à la symbolique païenne puis chrétienne ("our Crown . . . will . . a appear in all its Lustre and Splendor in a better [World]" [2.95]), qui conduit à confronter, par adjectifs interposés, mondes sublunaire et céleste, caractérisés par les traits traditionnels, dans "a poor momentary Trifle . . . before an Eternal and Immutable Crown of Glory!" (2.114) et dans les expressions "a poor transitory Earthly Diadem" (151) et "one of the brightest Crowns of Glory" (151). C'est la substance de la couronne, plus encore que sa place au sommet de la tête et que sa forme circulaire (emblème de perfection et de participation de la nature céleste), qui la distingue comme promesse de vie éternelle (voir Jacq 1.12, Pierre 4.5). Nulle allusion, en revanche, à la couronne christique hérissée de pointes, d'épines qui figurent les rayons solaires.

Nanties de tels atouts, les femmes deviendraient des exemples non pas figés mais animés d'une dynamique bienfaisante, comme une lumière (1.38) qui montrerait le bon chemin (2.141), réformerait autrui (150 où se retrouvent les sens de "glory"), voire sauverait une âme (la leur en premier lieu) de la mort éternelle (151) ${ }^{28}$ Plus ambitieux encore apparaît l'objectif astellien ultime: par leurs bonnes actions qui rediffusent, réfléchissent la lumière divine, les femmes inciteront autrui à glorifier Dieu ("to Let our Light so shine before Men, that they may see our Goodworks and Glorify our Father who is in Heaven" [172]). Elles constitueront des relais vers Sa gloire, la finalité étant Dieu et non soi (179). Devenue lumière, la croyante pourra à son tour éclairer autrui.

L'image de la lumière procure également à Astell le moyen de rappeler le véritable but de l'existence humaine, qui est la recherche de la vérité immuable - en fait, préparation déjà pour l'autre monde $(2.135$, 147). La lumière est omniprésente dans les figurations de Dieu et de la vérité, surtout dans la Partie 2, plus théorique et didactique. En accord avec la tradition, Dieu y est dépeint en Créateur de la vie (107), de l'univers et de l'homme (158). Il gouverne ce monde qu'Il a créé (160) et y est omnipotent $(1.9,2.174)$. Récurrent dans ces exemples, l'adjectif "all" souligne Sa toute-puissance (2.160) qui inclut, par périphrases,

28. "And perhaps the Glory of Reforming this Prophane and Profligate Age is reserv'd for you Ladies . . ." (2.150). "Glory" signifie "exalted renown, honourable fame," "resplendent majesty, beauty . . . effulgence of heavenly light" et aussi "circle of light round head or figure of saint, aureole, halo" $(O E D)$. 
omniprésence et omniscience. Sa perfection, toujours subordonnée à Son "infinitude," fait l'objet de mentions réitérées $(100,106,158,160)$.

Dieu est aussi lumière, en conformité avec les prologues de l'Évangile de saint Jean (1 Jean 1.5), et père de la lumière (2.91). ${ }^{29} \mathrm{~S}^{\prime} \mathrm{y}$ ajoute la métaphore de la source; source de lumière, Il est également source de vie (101), incarnée par le soleil (107). ${ }^{30}$ Astell se fait l'écho fidèle des psaumes $(117)^{31}$ et réfère aussi à Jésus, membre de la Trinité mentionnée quelques pages auparavant $(100,101)$, nommé ici "the Son of Syrac" (Ecclésiastiq. 23): "he who is The Light that Lightneth every one who comes in to the World" (117).

Lumière dans son être intime, par anthropomorphisme Sa face est de lumière, lumière voilée aux termes de la métaphore platonicienne: "these Clouds that hide the most adorable Face of GOD from us, these Mudwalls that enclose our Earthly Tabernacle and will not suffer us to be pierc'd with the Beams of his Glory" (2.160). Son visage réapparait peu après sous un autre vocable: "[the] Light of His Countenance" (167). ${ }^{32}$ Toutefois, n'est pas noté le vêtement de Dieu, une des manifestations les plus évidentes de $\mathrm{Sa}$ gloire, qui est la clarté rendant toutes choses visibles.

Dieu-lumière est lié à la vérité dont Il est la source, que la métaphore ("he who is . . . the Immutable Truth" [2.117]) soit introduite par une comparaison ("the Author of Life and Light is as inexhaustible a Source of Truth" [107]) ou non ("He who is the Fountain of Truth" [125]); Il la contient (106). Dieu conduira à la vérité tout chrétien qui le souhaitera (91, 105): Astell définit la nature de cette vérité et le moyen d'y atteindre.

Visée essentielle de l'être humain, la quête de la vérité se poursuit grâce à la raison octroyée par Dieu; la cultiver est un acte religieux, chez les femmes aussi. Même dissociée de la divinité, la vérité est inséparable de la lumière (2.141); les termes usuels, "bright" $(90,107)$ et "shine" $(90)$, sont accentués par "exceeding" (118). Elle est belle (2.116, 137, 142),

29. Voir le traité de John Norris (Two Treatises Concerning the Divine Light [London, 1692] 21, 23, 39, 53...) qui a aussi marqué Astell.

30. Voir Ellrodt 163 (n. 65): "Ficino's quotation from the Republic VI . . for the SunGod analogy was a mediaeval and Elizabethan as well as a Neoplatonic commonplace...."

31. Ps. 23.3, 35.4; Prov. 4.11, 8.20.

32. Cette expression se trouve sous la plume d'Astell à la dernière page des Letters Concerning the Love of God, between the Author of the Proposal to Ladies and Mr. John Norris: Wherein His Late Discourse, Shewing, That It Ought to Be Entire and Exclusive of All Other Loves, Is Further Cleared and Justified, 1695 (London, 1705) 180. 
attirante (141) et aimable (141, 142), mais Astell s'éloigne de la définition avancée par les platoniciens de la Renaissance. ${ }^{33}$

Toutefois, une multitude d'obstacles s'interposent entre la lumière divine de la vérité et l'âme. On relève une certaine variété dans leur figuration, fussent-ils de nature indéfinie $(1.9,2.85)$ ou précisée par le biais de la métaphore de l'écran et de ses avatars: nuage (ignorance [1.17, $21]$; ignorance et vanité $[2.97,107]$; bruits du monde [2.160]), vapeurs de la corruption (1.34), brumes (2.81), fumée des passions et des sens (11213), pellicule (125). Si l'image du voile n'est pas ici évoquée, en revanche le corps lui-même se fait écran (160), tout comme la matérialité, la vie du monde à partir de laquelle Astell construit de foisonnants réseaux de métaphores fondées sur la spatialité (extérieur-intérieur, surface-profondeur, ascension-chute) pour concrétiser l'antagonisme entre apparence et essence. Le rayonnement de la lumière intérieure est tributaire de ce qui entrave ou interdit sa diffusion (plutôt que son éclat) ou sa réception. Un écran, donc, empêche la femme de recevoir la lumière ou de diffuser la sienne, ce qui l'oblige à s'appuyer sur la raison pour trouver la vérité, pour dissiper les obstacles.

Au-delà de la description des attributs de la vérité, Astell s'attache à la nature de cette vérité dont elle distingue deux catégories: vérité humaine et vérité divine. La première est l'objet de l'entendement (2.92). Repris plus loin, ce point comporte le pluriel "all Truths" (100) et la référence aux limites de l'esprit humain $(100,105)$, qui introduisent indirectement une autre sorte de vérité, celle d'ordre religieux: la vérité de la révélation divine. Il importe ici de garder à l'esprit la double définition de la révélation donnée par Astell. ${ }^{34}$

La quête de la vérité s'exerce ainsi à deux niveaux non dissociés: "We shou'd search for Truth in our most abstracted Speculations, but it concerns us nearly to follow her close in what relates to the Conduct of our Lives" (2.120). Des voies différentes mèneront à ces deux vérités: soit la raison, soit la révélation. La vraie connaissance se trouve dans la lumière qui est Dieu, source de vérité et de connaissance. Astell réfère-telle aux voies traditionnelles de cette recherche? Elle ne renvoie pas à la révélation de la vérité par la vision de celle-ci qui est Dieu; certes, la nature de Dieu réside non dans l'obscurité mais dans la lumière (101); il n'y a pas de "dazzling darkness," comme chez Vaughan, mais une lumière éblouissante, sans rappel des visions plotiniennes exprimées par John

33. Sur eux, voir Ellrodt 35 et 89 (n. 172).

34. Vide supra $\mathrm{n} .12$. 
Smith. ${ }^{35}$ La vue de Dieu n'est pas accessible à l'individu qu'aveuglerait Sa lumière; comme dans la Bible, le face à face avec Dieu est impossible: "the Prospect it wou'd have given us wou'd have been too dazling [sic], too bright for Mortality to bear ..." (101). Néanmoins, Dieu a jugé bon de conduire à la vérité par Sa parole (158). En effet, la vraie lumière qui éclaire tout être humain est le Verbe de Dieu; c'est la vie de Dieu; elle l'éclaire de l'intérieur, lui permet de voir et de connaître, de mener toute sa vie dans la clarté.

L'illumination intérieure peut également guider vers la vérité. Pour Astell, comme pour saint Augustin, Dieu est la lumière infinie, source de toute vraie connaissance. ${ }^{36}$ Est-elle influencée par la doctrine augustinienne de l'illumination divine (illuminatio divina), fondée sur l'introspection et sur l'image de la lumière intérieure qui éclaire l'esprit (deux archétypes du [néo]platonisme)? Cette notion, au cœur de la logique interne qui réunit, chez saint Augustin, quatre idées distinctes mais étroitement liées, se prolonge chez Astell. Tout d'abord, en s'interrogeant, l'esprit peut découvrir la présence immédiate de Dieu en lui, "lumière immuable" qu'il peut voir grâce aux "yeux de l'âme" (2.125), cités chez Astell en relation avec la vision de l'éternité (88) et du "brillant et glorieux" royaume de Dieu (151). Cette lumière est celle de la raison (108) ou celle de la vérité introduite par une comparaison: "I consider, that as Light is always visible to us if we have an Organ to receive it, if we turn our Eyes towards it, and that nothing interpose between it and us; so is Truth, we are surrounded with it, and GOD has given us Faculties to receive it" (124). La lumière divine est le Créateur, on l'a vu (107). Elle est tenue pour la lumière de l'âme; l'immédiateté de la connaissance de Dieu renvoie à une approche ontologique, à l'épistémologie augustinienne. ${ }^{37} \mathrm{Le}$ deuxième trait de cette doctrine est la reconnaissance de la lumière divine comme lumière immuable de l'intellect, qui lui permet d'accéder à des vérités immuables. Par la reprise astellienne de la métaphore du soleil,

35. Voir Henry Vaughan, "The Night," Silex II, 1655, Henry Vaughan: The Complete Poems, ed. Alan Rudrum (Harmondsworth: Penguin, 1976) 290 (1.50): "There is in God (some say) / A deep, but dazzling darkness. . .." Une étude approfondie soulignerait des affinités entre Astell et Traherne ainsi que sa familiarité avec l'hermétisme et avec les images empruntées à l'alchimie.

36. Augustine, Confessions, c. 400, trans. Rex Warner (New York: New American Library, 1963) 7.10.149: "He who knows truth knows that light, and he who knows that light knows eternity."

37. Elle est différente de l'approche thomiste ou "cosmologique" qui souligne la médiation de la connaissance de Dieu par les sens. Voir Robert L. Fastiggi, "The Divine Light Within: Reflections on the Education of the Mind to God in Augustine, Anselm, Bonaventure and Newman," Faith Seeking Understanding: Learning and the Catholic Tradition, ed. George C. Berthold (Manchester, NH: Saint Anselm College P, 1991) 197. 
source de lumière physique qui rend toutes choses visibles, Dieu est la source de lumière spirituelle (107). Dieu est à notre esprit ce que le soleil est à notre vue (124); le soleil est source de lumière, Dieu de vérité (125). ${ }^{38}$ On ne trouve pas trace du troisième trait dans la Proposal. Pour saint Augustin, Dieu existe dans le tréfonds de l'âme et l'individu prend conscience qu'une part de lui-même le transcende. Il la situe dans la mémoire, profondeur intérieure ou bien issue vers les étendues illimitées de l'infini. L'esprit est un abîme qui s'ouvre à la lumière infinie du divin. Il en va de même pour le dernier trait: l'âme est image de Dieu, de la Trinité; les Confessions (livre 13) suggèrent que la triple réalité de l'esprit, en tant qu'existence, entendement et volonté, aide à la compréhension d'un Dieu en trois personnes. Astell associe volonté et entendement, les définissant comme complémentaires $(1.17,2.83,85,115$, 153), mais elle ne partage pas la méditation augustinienne qui voit en la vie de l'âme le miroir de l'essence trinitaire de Dieu.

La lumière intérieure qui illumine l'esprit est, selon Astell, la raison. Dans le cas de la vérité humaine, chacun peut la rechercher grâce à sa raison: "Truth is so near at hand . . . in our own Breasts" (2.118); il s'agit là des implications du cogito cartésien: "every one shou'd have a Teacher in their own Bosoms" (118). Quant à la vérité divine, sa quête impose à la raison, si elle est bien formée, d'être aidée par la révélation; raison et révélation ne s'excluent pas, au contraire. ${ }^{39}$ Dans la poursuite de la vérité, l'entendement mène à la lumière ("Our Understandings are sufficiently illuminated to lead us to the Fountain of Life and Light" [101]), et il est à son tour "illuminé par la connaissance de la vérité" (1.38). ${ }^{40}$

Dieu peut conduire à la vérité par l'autre voie qu'est l'amour. Dans son désir de voir l'individu touché par la lumière divine, Astell renverse l'image de la blessure mortelle causée par une flèche, ici de lumière: "to be pierc'd with the Beams of his Glory, and wounded, not to Death but Life, with the Arrows of his Love and Beauty" (2.160). Il ne mourra pas mais vivra grâce à la blessure; en arrière-plan se dessine la couronne de la passion dont les épines blessent le Christ; mais cette passion rachète l'humanité et, par là-même, donne la vie.

38. "illuminated with the knowledge of the Truth" (1.38). Voir Ellrodt 163 (n. 65): "Like St Augustine the Renaissance Platonists attributed a metaphysical function to divine light; it bestowed upon the human mind the power to think as the sun bestowed upon the eyes the power to see...."

39. Vide supra $\mathrm{n} .12$.

40. 1.38: "whose Understanding being clearly illuminated with the knowledge of the Truth. .. ." Voir aussi 2.81: "so Vice that spawn of the Devil, that Ignis fatuus which can't subsist but in the dark night of Ignorance, casts forth Vapours and Mists to darken the Soul and eclipse the clear light of Knowledge from her View." 
Dieu est aussi objet d'amour: "the only Amiable Being, who is altogether Lovely and worthy of All our Love, the Object of our Hope, the Sum of our Desire, the Crown of our Joy . . ." (2.160). Astell adhère, comme Norris, à la croyance platonicienne de l'unité, qui conçoit Dieu comme la source de toute chose et, à ce titre, comme le seul objet approprié à l'amour humain (106). L'objet de l'amour de l'homme ne peut être que Dieu; la forme de la construction interrogative le souligne dans la question: "What can be the object of Love but . . . the Image of the Deity impress'd upon a generous and God-like Mind . . ." (1.49). Cet amour exclusif, difficile à accepter pour Astell, est au cœur de sa correspondance avec Norris, publiée sous le titre Letters Concerning the Love of God (1695).

De plus, l'amour pour Dieu conduit à la vérité. Norris y écrit dans la Préface: "An affectionate Sense of GOD will discover more of him to us, than all the dry study and speculation of Scholastick Heads"; il ajoute: "The Fire in our Hearts will give the best and truest Light to our Eyes" (Letters n.p.). Le feu symbolise l'ardeur de cet amour (tandis que la lumière demeure intellectuelle et spirituelle), dans la ligne des platoniciens de Cambridge pour qui la vision de Dieu est le privilège des âmes aimant d'un amour fervent. ${ }^{41}$

Source de lumière, Dieu est également source inépuisable de joie et de bonheur (2.107). Aimer Dieu inonde du plus grand des bonheurs possibles (170). Une précision est apportée: le bon chrétien sera plus heureux encore dans l'autre monde où l'âme pourra contempler et jouir de Dieu $(100,171)$. C'est en des termes passionnés - ton différent de celui de la Proposal - qu'Astell loue la ferveur religieuse de la dédicataire des Letters Concerning the Love of God, Lady Catherine Jones, en reprenant le symbole du feu utilisé par Norris: "where can a Discourse of the Love of GOD be more appositely presented than to a Soul that constantly and brightly shines with these Celestial flames?" $\left(\mathrm{A} 6^{\mathrm{v}}\right)$. La métaphore est filée pour en qualifier la dévotion: "such a serious, reverent and unaffected Devotion . . . so equally composed of Heat and Light as gave me a lively Idea of Apostolical Piety, and made me every time I prayed her, fancy my self in the Neighbourhood of Seraphick Flames!" (A $7^{\mathrm{v}}$ ). L'intensité de cet amour proche du ravissement est communicative, traduite en des termes plus denses que ceux des contemporains.

41. Voir Patrides 13-14 et Basil Willey, "Rational Theology: The Cambridge Platonists," The Seventeenth-Century Background: Studies in the Thought of the Age in Relation to Poetry and Religion, 1934 (London: Routledge, 1979) 125. 
En habile stratège, Astell ouvre et clôt la Proposal sur la notion de bonheur. Elle précise, d'une part: "My earnest desire is, That you Ladies, would be as perfect and happy as 'tis possible to be in this imperfect state ..." (1.8), et, d'autre part: "Happiness is the natural Effect as well as the Reward of an ardent Love to GOD and what necessarily flows from it, Universal Piety" (2.167). L'auteur joue sur l'intérêt des lectrices (souligné dans le titre) qu'elle incite, par le biais de leur vanité et de leur ambition, à poursuivre des buts plus élevés, à s'amender, à se conformer à la volonté divine qui mène non seulement au salut mais aussi au bonheur à découvrir en soi (171).

Le devoir du chrétien, ou, plutôt ici, de la chrétienne, est d'aimer Dieu, d'accomplir Sa volonté, de vivre pleinement sa nature humaine et de faire du prosélytisme. Astell s'assigne pour mission d'enseigner aux femmes à penser clairement, à l'aide de ses propres écrits rédigés euxmêmes avec clarté: "The Method of Thinking" et "good Speaking and Writing" (2.139) se complètent.

Aimer Dieu équivaut à réaliser Sa volonté (2.171). Accomplir la volonté divine signifie accomplir la sienne propre (156); la croyante demande à Dieu de la guider dans cette voie (161), question déjà formulée: "what must I do to fill up my Vacuities, to accomplish my Nature?" (83). "Vacuities" est à "retourner" en potentialité, en capacité à accueillir, à remplir, comme le suggère la réponse: "The business of our Lives will be to improve our Minds and to stretch our Faculties to their utmost extent, that so we may have the fullest enjoyment our Nature will admit, of this ever satisfying and yet ever desirable, because an Infinite, and our True, Good" (161). ${ }^{42}$ L'auteur indique le but poursuivi et le moyen d'y parvenir: la chrétienne doit vivre pleinement la dimension humaine de son existence - ce qui reprend la Partie 1 (46) - en cultivant sa raison et en exploitant les talents reçus de Dieu $(2.148,156)$, tendus vers la recherche de la vérité.

Intervient ici la tâche que s'attribue Astell: augmenter l'aura lumineuse des femmes et, par là, amplifier le lustre de leur exemple qui influencera davantage encore les autres et servira à louer Dieu ainsi qu'à rendre les chrétiens plus heureux encore ("Has our Boutiful Lord set no limits to our Happiness but the Capacity of our Nature, and shall we set less, and not shine to extend our Capacities to their utmost reach?"

42. "to improve our Minds and to stretch our Faculties to their utmost extent" (2.161) fait écho à "they might enlarge their prospect, rectify their false Ideas, form in their Minds adequate conceptions of the End and Dignity of their Natures" (2.78). 
[2.88]). Dans 1"'Introduction" de la Partie 2, à la fois résumé et perspective, elle entreprend de stimuler ses lectrices: "I suppose then that you're fill'd with a laudable Ambition to brighten and enlarge your Souls, that the Beauty of your Bodies is but a secondary care . . ." (75); au début de la Partie 2, elle réunit les verbes "brighten" et "shine" dans "[understanding] will enlarge . . . will brighten and shine forth with diffusive Rays" (83).

Dans ces trois cas, le réseau des images lumineuses se double d'un tissu d'images spatiales ("limits," "extend," "reach," "enlarge"), issu, en général, de la racine latine capax ("qui peut contenir") venant de capere ("contenir, être susceptible de"). De capabilis dérive "capable" ("susceptible; having the power or fitness for; wicked enough for; gifted, able"), et de capacitas "capacity" ("holding-power, receiving-power; mental power, faculty, capability, opportunity" [OED]). Cette terminologie récurrente dans la Proposal appartient à une logique d'antithèses: d'un côté, une dynamique positive soutenue par des images d'accroissement, d'intensification de la lumière, d'élargissement, d'expansion, d'élévation; de l'autre, un mouvement négatif traduit par un ensemble de métaphores spatiales articulées autour de thèmes tels qu'obscurcissement, obstacles, rétraction, contraction, chute. L'analyse minutieuse de ces métaphores livre des résultats très significatifs. Clarté et expansion, par exemple, fonctionnent en binôme quand il importe d'exploiter les capacités de l'entendement à conduire à la lumière: "the perfection of the Understanding consisting in the Clearness and Largeness of its view, it improves proportionably as its Ideas become Clearer and more Extensive" (2.99).

Le dessein d'Astell est d'entraîner les femmes à une réflexion indépendante; rhétoricienne avisée, elle manie l'ambivalence du substantif "reflection" pour passer du concret à l'abstrait, de ce que connaissent bien les femmes à ce qu'elle veut leur faire découvrir; il s'agit de les mener du superficiel à l'essentiel, de la réflexion du miroir à celle de l'esprit, propos qui ouvre la Proposal: "Your Glass will not do you half so much service as a serious reflection on your own Minds" (1.6). L'argument est repris, en 1697, sous forme de question induisant une réponse affirmative: "Were not a Morning more advantageously spent at a Book than at a Looking Glass, and an Evening in Meditation than in Gaming?" (2.151). Précaution est prise: nul(le) ne doit se méprendre sur la nature de l'étude suggérée, qui n'est point de briguer l'érudition ("this is the Knowledge that pufeth up" [97]), référant à saint Paul sans le citer (1 Cor. 8.1); cette distinction entre vrai et faux savoir s'inscrit dans la condamnation 
astellienne des érudites. ${ }^{43}$ Une fois encore, l'important est de détourner l'esprit du clinquant des apparences pour l'orienter vers la profondeur de l'essence, fonction assurée par la réunion de "tinsel ware" et de "false Light" (1.12), de "false reasoning" et de "glittering Non-sense" (2.75). Clinquant, doré (et non "en or") ${ }^{44}$ représentent l'une des facettes de l'opposition entre nature et artifice, entre naturel et maquillage, adaptation judicieuse des images au lectorat concerné, quand il s'agit d'évoquer le contenu de sa méthode. ${ }^{45}$

L'auteur établit un parallèle entre fond et forme, entre méthodes de réflexion et méthodes d'expression: "The Method of Thinking has been already shewn, and the same is to be observ'd in Writing . . ." (2.139). Elle poursuit le double but d'enseigner à penser avec clarté ("Clear, Rational and Concluding [Arguments]. . . A And I hope the Reader will receive from hence more light into the way of Arguing . . ." [131]) et de s'exprimer de même. L'obscurité du fond et de la forme est maintes fois dénoncée comme indice de dissimulation et, donc, de tromperie. ${ }^{46}$ "Equivocal Terms," "Sophisms" (133) et "Fallacious Reasonings" (135) se voient rejetés sans appel. En revanche, la clarté structurelle et formelle est de bon augure: "Scarce any thing conduces more to Clearness, the great Beauty of writing, than Exactness of Method" (138).

La Partie 2 fournit un ensemble de règles pour raisonner avec rigueur, clarté et logique, très inspiré des quatre règles exposées dans la

43. Voir 1.23-24, 2.76 et 147, ainsi que les expressions "Female Virtuoso" (1.22) et "walking libraries" (2.180). Toutes deux pourraient faire écho à No Fools like Wits; or, The Female Virtuosoes, adaptation partielle des Femmes savantes que réalisa Thomas Wright et qui porte plusieurs sous-titres: No Fools like Wits; or, The Female Virtuosoes; or, The Refusal; or, The Ladies Philosophy (1693 [London, 1721]). Sir Maurice Meanwell y affuble son épouse savante, véritable virago, des qualificatifs suivants: "my walking University, my puzzling Library of Flesh" (2.1.24). À l'endroit des érudites, Astell n'use pas d'image de lumière à la manière de Bathsua Makin qui mêle astronomie, superstition et misogynie dans "A learned woman is thought to be a comet that bodes mischief whenever it appears" (An Essay to Revive the Ancient Education of Gentlewomen, in Religion, Manners, Arts, \& Tongues, with an Answer to the Objections against This Way of Education, 1673, Bathsua Makin, Woman of Learning, ed. Frances Teague [Lewisburg: Bucknell UP, 1998] 109).

44. La connotation péjorative de ces images ("gilt" ou "gilded") se retrouve dans Some Reflections on Marriage, Occasion'd by the Duke \& Dutchess of Mazarine's Case; Which Is Also Consider'd, 1700, Astell: Political Writings, ed. Springborg (Cambridge: Cambridge UP, 1996) 49, 60, 74.

45. La même palette d'images est utilisée pour définir la véritable éloquence (2.137).

46. "Obscurity, one of the greatest faults in Writing . . . is sometimes design'd, to conceal an erroneous opinion which an Author dares not openly own, or which if it be discover's he has a mind to evade" (2.138); le lexique de la dissimulation et de la révélation est aussi présent dans 2.133 et dans 2.143 . 
"Deuxième partie" du Discours de la méthode ${ }^{47}$ et, surtout, dans La Logique, ou L'Art de penser (1662) de Pierre Nicole et Antoine Arnauld, auquel elle réfère sans le nommer. ${ }^{48}$ Six "règles" (2.126-28) numérotées avec soin sont énoncées (alors que l'ouvrage originel n'en comporte que cinq...): définition du sujet de réflexion, concentration sur l'essentiel, ordre croissant de complexité dans l'analyse, exhaustivité dans le traitement du sujet ("to Consider All that can let in Light" [127]), concentration sur lui ("carrying Light and Evidence in ev'ry step we take" [127]), enfin, reflet quasi littéral de la première règle cartésienne: "To judge no further than we Perceive, and not to take any thing for Truth, which we do not evidently Know to be so" (128). ${ }^{49}$

L'expression, quant à elle, est soumise à la même exigence de rigueur et de limpidité: $:^{50}$ "our Expressions must be more plain and explicit . . ." (2.138). ${ }^{51}$ Sa simplicité est garante de la qualité de la pensée: "For Plain and Significant Language is ever best, we have a mistaken Idea of Learning if we think to pretend to't by sending our Reader every minute to the Dictionary" (146), ce qu'illustre la métaphore de l'amande et de sa coque: "the Readers [must] not lose the pleasure of the Kernel, by the pain they find in cracking the Shell" (144). Préoccupée de cerner l'essence de la rhétorique, Astell cite Arnauld et Nicole: "all that is useful in this Art [Rhetoric], 'is the avoiding certain evil ways of Writing and Speaking, and above all an Artificial and Rhetorical Stile Compos'd of false Thoughts, Hyperboles and forc'd Figures which is the greatest Fault in Rhetoric"' (137). ${ }^{52}$ Ce procès dressé, elle livre sa définition de la vraie rhétorique: "the design of Rhetoric is to remove those Prejudices that lie in the way of Truth . . . to place our Subject in a Right Light . . ." (137).

47. Descartes, non nommé, est qualifié de "a Celebrated Author" (2.123) quand elle cite un extrait des Principes de la philosophie [voir Descartes 453 (Partie 1, § 45)] où l'adjectif "clear" et la métaphore de l'œil de l'esprit sont récurrents.

48. Elle le décrit ainsi: "a very Judicious Writer on this Subject (to whose Ingenious Remarks and Rules I am much obliged)" (2.117; voir aussi 2.137). Astell s'inspire de la traduction anglaise de 1693 plutôt que de celle de 1674. Ces règles, indique Arnauld, sont empruntées à un opuscule intitulé De l'esprit géométrique et de l'art de persuader que rédigea Pascal après son entrée à Port-Royal, vers 1658 .

49. Voir Descartes, Discours de la méthode, 1637, Euvres et lettres 103-04. Dans sa discussion des règles gouvernant le syllogisme (2.131-34) Astell suit aussi Arnauld et Nicole (Logic; or, The Art of Thinking, 1674 [London, 1693] 3.2-10.215-382).

50. Une étude des métaphores liquides dans la Proposal serait riche d'enseignements.

51. Voir aussi "to make a shew of Wit by tedious Arguings and unnecessary Flourishes, does only Perplex and Incumber the Matter, Intuition being the Simplest, and on that account the best way of Knowing" (2.126).

52. The Art of Speaking (1676), rédigé par l'oratorien Bernard Lamy (1640-1715) qui suivit le programme de Port-Royal sur la rhétorique, est qualifié de "an Ingenious Treatise" (2.144). 
Écrire avec clarté, souligne Astell, exige de prêter attention à l'orthographe (2.143), à la grammaire (144) et au style (144-47). En ce dernier domaine, le texte est précis; le principe directeur est de suivre la nature:

as Thinking conformably to the Nature of Things is True Knowledge, so th'expressing our Thoughts in such a way ... and with greatest Clearness and Life . . . is the best Eloquence. For if our Idea be conformable to the Nature of the thing it represents, and its Relations duly stated, this is the most effectual Way both to Inform and Persuade. . . (142)

Le style ne doit être ni mal dégrossi ni rustre (143), sous peine d'être rébarbatif et de faire fuir le lecteur (145). Il faut, en outre, se méfier de l'imagination et des passions; ce qui pose la distinction entre convaincre et persuader: "Whilst th'exactness of his Method, and Force of his Reason Enlighten and Convince the Mind; the Vivacity of his Imagination and insinuating Address, gain the Affections ..." (145). Suivent des conseils pour trouver le juste milieu: "without Dryness [sic] or Obscurity, and Florid enough to entertain th'Imagination without Distracting the Mind" (146). Là aussi la clarté est de mise

La Proposal met en pratique les propres recommandations de l'auteur. La Partie 1 s'applique à convaincre et à persuader, la Partie 2 à instruire, à argumenter et à expliquer (deux fois plus longue, elle comporte quatre chapitres). Sa maîtrise de la rhétorique ressort de son choix des arguments (inventio) adaptés à son lectorat, de leur arrangement (dispositio) et de son style (elocutio). À la captatio benevolentiae succèdent la proposition (qui n'intervient qu'au tiers de la Partie 1), l'analyse des avantages (confirmatio) et les réponses à des objections potentielles (refutatio). ${ }^{53}$ Les compliments, formulés par Norris à l'endroit du style astellien des Letters Concerning the Love of God, s'appliquent tout à fait à la Proposal:

so admirable both your Thoughts and Expressions upon it [Subject], such Choiceness of Matter, such Weight of Sense, such Art and Order of Contrivance, such Clearness and Strength of Reasoning, such Beauty of Language, such Address of Stile, such bright and lively Images and Colours of Things, and such moving strains of the most natural and powerful Oratory.... (A $\left.8^{\mathrm{r}}\right)$

La chaleur du style ("such warm quickning Discourses" [A9 $\left.{ }^{\mathrm{r}}\right]$ ) doit réveiller l'ardeur des lecteurs à l'égard de Dieu. Choix des arguments,

53. Sur cet aspect, voir Christine Mason Sutherland, "Mary Astell: Reclaiming Rhetorica in the Seventeenth Century," Reclaiming Rhetorica: Women in the Rhetorical Tradition, ed. Andrea A. Lunsford and James J. Murphy (Pittsburgh: U of Pittsburgh P, 1995) 93-116. 
organisation, clarté du raisonnement, constructions symétriques des phrases, images éclatantes caractérisent, dans ses divers écrits, son art consommé de la rhétorique.

L'existence même de cet ouvrage et son succès lors de sa parution illustrent le pouvoir de la raison et du langage, exercé par une femme, réalisant ainsi le vœu d'Astell de donner droit de cité au discours des femmes dans le cadre de la société patriarcale, en insistant sur son utilité morale pour la communauté. En effet, rhétorique et éthique ne sont pas dissociées: "For it is to little purpose to Think well and speak well, unless we Live well, this is our Great Affair and truest Excellency. . . . And all her [a woman's] Eloquence is but an empty noise, who employs it in any other design than in gaining Proselytes to Heaven" (2.147). Rhétorique et religion se rejoignent même: "the way to be good Orators is to be good Christians" (142). Astell place la lumière de l'esprit et de l'expression au service de la morale chrétienne et met en question la classique métaphore guerrière de la rhétorique victorieuse d'un ennemi (souvent l'auditoire qui doit être vaincu): "Truth not Victory is what we shou'd contend for in all Disputes ... And therefore we pervert our Reason when we make it the Instrument of an Endless Contention ..." (132-33). À travers Port-Royal, Astell suit saint Augustin pour qui le principe rhétorique fondamental est l'amour. $^{54}$

Mary Astell aborde sa Proposal et termine sa Préface des Letters Concerning the Love of God selon une technique semblable: faire miroiter aux femmes la double promesse d'une beauté durable et du bonheur. Elle définit ainsi sa Proposal: "[its] only design is to improve your Charms and heighten your Value, by suffering you no longer to be cheap and contemptible. Its aim is to fix that Beauty, to make it lasting and permanent, which Nature with all the helps of Art cannot secure" (1.5). Un an plus tard, l'argument reste identique: "that Ladies may be at last convinced that the Beauty of the Mind is the most charming Amiableness, because most lasting and most divine, and that no Ornaments are so becoming to a Lady as the Robe of Righteousness and the Jewels of Piety" $\left(A 7^{v}\right)$. Vêtement, bijou et beauté éclatante ne participent plus désormais de la panoplie de la femme futile mais des attributs de la chrétienne.

Les parures changent de registre et ne concernent plus le corps mais l'âme $(1.19,21,24,36)$, dans le souci de stimuler et d'exhorter l'ambition

54. Voir Sutherland, "Love as Rhetorical Principle: The Relationship between Content and Style in the Rhetorick of St. Augustine," Grace, Politics and Desire, ed. Hugo A. Meynell (Calgary: U of Calgary P, 1990) 139-54. 
féminine à des fins plus nobles: "I suppose then that you're fill'd with a laudable Ambition to brighten and enlarge your Souls. . . Books are now become the finest Ornaments of your closets and Contemplation the most agreeable Entertainment of your leisure hours" (2.75). Le propos rejoint celui des Letters: "to excite a generous Emulation in my Sex, perswade [sic] them to leave their insignificant Pursuits for Employments worthy of them" $\left(\mathrm{A} 6^{1}\right){ }^{55}$ Pour mieux encore se présenter comme un modèle enviable, un exemple à imiter, elle recourt à un lexique propre à évoquer une quasi-sensualité du bien et des joies issus de l'étude (1.20, 33, 2.157-158, 167); ${ }^{56}$ ainsi parle-t-elle d'épicurisme, au sens édulcoré $(1.26,2.168) .{ }^{57}$

Tout au long de ses essais, Astell élabore un appareil d'images centrées sur la lumière, également présent dans ses poèmes religieux encore inexplorés et dans ses Letters. Les uns et les autres obéissent au même impératif de clarté, imposé au fond et à la forme, que son opus magnum, The Christian Religion as Profess'd by a Daughter of the Church (1705), rédigé lors de sa participation aux débats sur la tolérance religieuse. Parce que la raison, principe éclairant, confère à l'individu sa dimension à la fois humaine et divine, l'auteur démontre la pertinence et la primauté, chez les femmes, du développement de leurs facultés rationnelles et de leur éducation, qui fera d'elles les êtres autonomes voulus et créés par Dieu. ${ }^{58} \mathrm{Sa}$ démarche réussit à concilier, en une adroite synthèse, l'amour pour Dieu, le rationalisme, son ambition personnelle et l'émancipation des femmes.

\section{Guyonne LEDUC}

Université Charles de Gaulle - Lille III

55. Les mêmes termes apparaissent déjà dans 1.5: "Wou'd excite in you a generous Emulation to excel in the best things. ..."

56. Voir par exemple 2.157: "a Divine Sensation gives us a relish of what's Good. ..." et 2.167: "how pure and exalted is that Pleasure, how highly entertaining, which results from the right use of our Faculties, and Fruition of the Sovereign Good! . . . She tasts [sic] a Pleasure which the World can neither give nor take away, nor can Worldly Minds so much as Imagine it. ..."

57. Voir 1.26: "'Tis in truth the highest Epicurism . . ." et 2.168: "Good Christians being indeed the truest Epicures, because they have the most tastful and highest Enjoyment of the greatest Good." Goûter aussi 1.27: "No perfume will be thought so grateful as the Odour of Good Works...."

58. Voir Catherine Gallagher, "Embracing the Absolute: The Politics of the Female Subject in Seventeenth-Century England," Genders 1 (1988): 34 et Alessa Johns, "Mary Astell's 'Excited Needles': Theorizing Feminist Utopia in Seventeenth-Century England," Utopian Studies 7.1 (1996): 62-63. 